\title{
Industry Collaboration and Primary Guest Authorship of High-Impact Randomized Clinical Trials: A Cross-Sectional Study
}

\author{
Nitin Roper, M.D. ${ }^{1}$ and Deborah Korenstein, M.D. ${ }^{2}$ \\ 'Weill Cornell Medical College, New York, NY, USA; ${ }^{2}$ Department of Medicine, Memorial Sloan Kettering Cancer Center, New York, NY, USA.
}

BACKGROUND: Journals have increased disclosure requirements in recent years, in part to deter guest authorship. The prevalence of guest authorship among primary authors (first and last) in the current era of increased disclosure requirements is unknown.

OBJECTIVES: Our aim was to examine the self-reported prevalence of guest authorship among primary authors from a sample of randomized clinical trials with and without industry funding and industry collaboration in the design, analysis or reporting of trials.

DESIGN: Cross-sectional analysis of randomized, drug/ device clinical trials with published details on the "Role of the Funding Source/Sponsor" published in highimpact biomedical journals between 1 December 2011 and 31 November 2012. Phase 1 or 2 trials, secondary trial analyses, and trials that were not listed on ClinicalTrials.gov were excluded. Primary guest authorship was defined, based on International Committee of Medical Journal Editors (ICMJE) criteria, when neither the first nor last author contributed to either of the following: 1) the design of the trial or the analysis/ interpretation of data; or 2) drafting part or all of the manuscript.

PARTICIPANTS: One hundred and sixty-eight randomized clinical trials that met inclusion criteria were included.

MAIN OUTCOME MEASURES: We measured differences in the prevalence of guest authorship between trials with neither industry funding nor collaboration and 1) trials with industry funding without collaboration, and 2) trials with industry funding with collaboration.

RESULTS: The overall prevalence of primary guest authorship was $6 \%$ (10/168). Primary guest authorship was significantly more common in trials with industry funding with collaboration than in those with neither industry funding nor collaboration [13.2\% (10/76) vs. $0 \%$ (0/39); $p<0.02]$. Primary guest authorship did not differ between trials with industry funding without collaboration and trials with neither industry funding nor collaboration.

CONCLUSIONS: Among a sample of randomized, drug/ device clinical trials in high-impact biomedical journals, primary guest authorship was overall uncommon and occurred exclusively among trials with industry funding with collaboration.

Received December 23, 2014

Revised March 3, 2015

Accepted March 6, 2015

Published online April 2, 2015
KEY WORDS: conflict of interest; industry funding; industry collaboration; guest authorship; honorary authorship; primary guest authorship.

J Gen Intern Med 30(10): 1421-5

DOI: $10.1007 /$ s11606-015-3299-1

(C) Society of General Internal Medicine 2015

\section{INTRODUCTION}

Guest authorship, defined as attribution of research article authorship to investigators not meeting standard authorship criteria, has been a concern to the medical community because of the importance of honesty and accountability to the integrity of scientific research and the potential for legal ramifications. ${ }^{1}$ Since multiple studies have estimated the prevalence of guest authorship to be 17 to $41 \%$ in the medical literature, ${ }^{2-5}$ several interventions have been implemented to increase transparency and reduce misrepresentation of clinical research. Many journals, based on International Committee Medical Journal Editors (ICMJE) recommendations, ${ }^{6}$ require sponsors and authors to detail their specific role in the analysis/interpretation of data, design of the study, and writing or revision of the manuscript. Authors are also required to disclose industry payments and all potential conflicts of interest. Clinical trials are required to be listed on an online registry, which in the U.S. (on ClinicalTrials.gov) includes identifying the principal investigator(s). ${ }^{7}$

One survey estimated that the prevalence of guest authorship, among any study author, in top general medical journals decreased after these interventions from $29 \%$ in 1996 to $21 \%$ in 2008. ${ }^{5}$ However, given that primary (first and last) authors are the authors most likely to determine study content, guest authorship of these authors may be most important. Since litigation related to rofecoxib revealed direct evidence of guest authorship among first authors of clinical trials sponsored by industry, ${ }^{8}$ no studies, to our knowledge, have examined the prevalence of guest authorship among primary authors of industry sponsored studies.

The objective of this study was to examine the current prevalence of guest authorship among primary (first and last) authors, based on self-reporting, from a sample of randomized clinical trials published in high-impact journals. We limited our analysis to trials listed on ClinicalTrials.gov to determine the prevalence of guest authorship with adherence to optimal standards of transparency. We stratified trials by industry collaboration in the design, analysis, or reporting and by funding status, 
given recent evidence that collaboration may be a better predictor of clinical trial bias than funding alone. ${ }^{9}$ We hypothesized that guest authorship would be more common among trials with industry collaboration in the design, analysis, or reporting of results than among other trials, and that trials with guest authorship would be less likely to identify a primary author as a principal investigator on ClinicalTrials.gov.

\section{METHODS Study Inclusion/Exclusion Criteria}

We identified all randomized clinical trials of drugs and devices published between 1 December 2011 and 31 November 2012 in biomedical journals for which Journal Citation Reports 2012 reported an impact factor greater than 11 and that published details on the "Role of the Funding Source/Sponsor." The following journals were included: American Journal of Respiratory Critical Care, Annals of Internal Medicine, British Medical Journal (BMJ), Journal of the American Medical Association (JAMA), Lancet, Lancet Infectious Disease, Lancet Neurology, Lancet Oncology, New England Journal of Medicine (NEJM), and Public Library of Science (PLoS) Medicine. We excluded phase 1 or 2 trials, secondary trial analyses, and trials that were not listed on ClinicalTrials.gov.

\section{Definitions}

We determined funding based on information in or accompanying each publication. We categorized trials as having industry funding with collaboration when any for-profit organization funded the trial and had any role in its design, analysis or reporting; as having industry funding without collaboration when any for-profit organization funded the trial, but had no role in its design, analysis or reporting; or as having neither industry funding nor collaboration.

Based on ICMJE criteria, we defined a trial as having primary guest authorship when neither the first nor the last author contributed to either of the following: 1) the design of the trial or the analysis/interpretation of data, or 2) drafting part or all of the manuscript. If either a first or last author participated in the design/analysis/interpretation or in the drafting of the manuscript of a trial, then such a trial was not considered to be primary guest authored. Previous studies ${ }^{2-5}$ similarly used ICMJE criteria to define guest authorship among any study author; however, unlike previous studies, we did not include revising the manuscript "critically for important intellectual requirement" as a contribution, since we sought to focus on the initial writing of the manuscript, which is generally viewed as the responsibility of the primary authors. ${ }^{10}$

\section{Data Extraction}

Two investigators (NR and DK) independently assessed first and last author contribution to the design of the trial, analysis/ interpretation of the data, and drafting of the manuscript, based on author self-report, in the main publication or any appendices. Authorship contribution was recorded as "absent" if there was an explicit statement of contribution only by individuals other than the primary authors. We excluded trials for which no authorship contribution could be determined from further analysis (Fig. 1). All differences between investigators were resolved by consensus. Inter-rater agreement for trial variables was high $(\mathrm{K}=0.80)$. One investigator $(\mathrm{NR})$ recorded funding (industry and/or government/non-profit), and industry collaboration. Academic affiliation of first and last study authors was also noted. For each trial, we extracted the principal investigator(s) identified on ClinicalTrials.gov. Information on payments given to a primary author from the sponsor was obtained from ICMJE disclosure forms.

\section{Statistical Analysis}

We conducted two sets of analyses, comparing the prevalence of guest authorship and other variables between trials with neither industry funding nor collaboration and 1) trials with industry funding with collaboration and 2) trials with industry funding without collaboration, using Fisher exact tests. Statistical analyses were conducted using SAS version 9.3 (SAS Institute Inc.). All p values were two-tailed, with significance defined as $p<0.05$.

\section{RESULTS}

There were 168 trials from ten high-impact journals describing the results of drug and device trials included in our analysis (Fig. 1). Thirty-nine trials ( $23 \%)$ had neither industry funding nor industry collaboration, 76 (45\%) had industry funding with industry collaboration, and $54(32 \%)$ had industry funding without collaboration (Table 1).

The overall prevalence of primary guest authorship was $6 \%(10 / 168)$. Primary guest authorship was significantly more common in trials with industry funding with collaboration than in those with neither industry funding nor collaboration [13.2\% $(10 / 76)$ vs. $0 \%(0 / 39) ; p<0.02]$ (Table 1). Primary guest authorship did not differ between trials with industry funding without collaboration and trials with neither industry funding nor collaboration (Table 1). The prevalence of individual components of guest authorship were similar in trials with industry funding with collaboration and those with neither funding nor collaboration, including involvement of neither primary author in drafting the manuscript $[9.2 \%(7 / 76)$ vs. $0 \%(0 / 45) ; p=0.09]$ and involvement of neither primary author in designing the trial or analyzing/interpreting data $[3.9 \%(3 / 76)$ vs. $0 \%(0 / 45)$; $p=0.55$ ] (Table 1).

Trials with industry funding with collaboration were less likely than trials with neither industry funding nor collaboration to identify the first or last author as a principal investigator on ClinicalTrials.gov [25.0 \% (19/76) vs. $86.7 \%(39 / 45)$; $p<0.001]$ (Table 2). All first authors and approximately $90 \%$ 
18 Medical Journals with Impact Factor $>11$

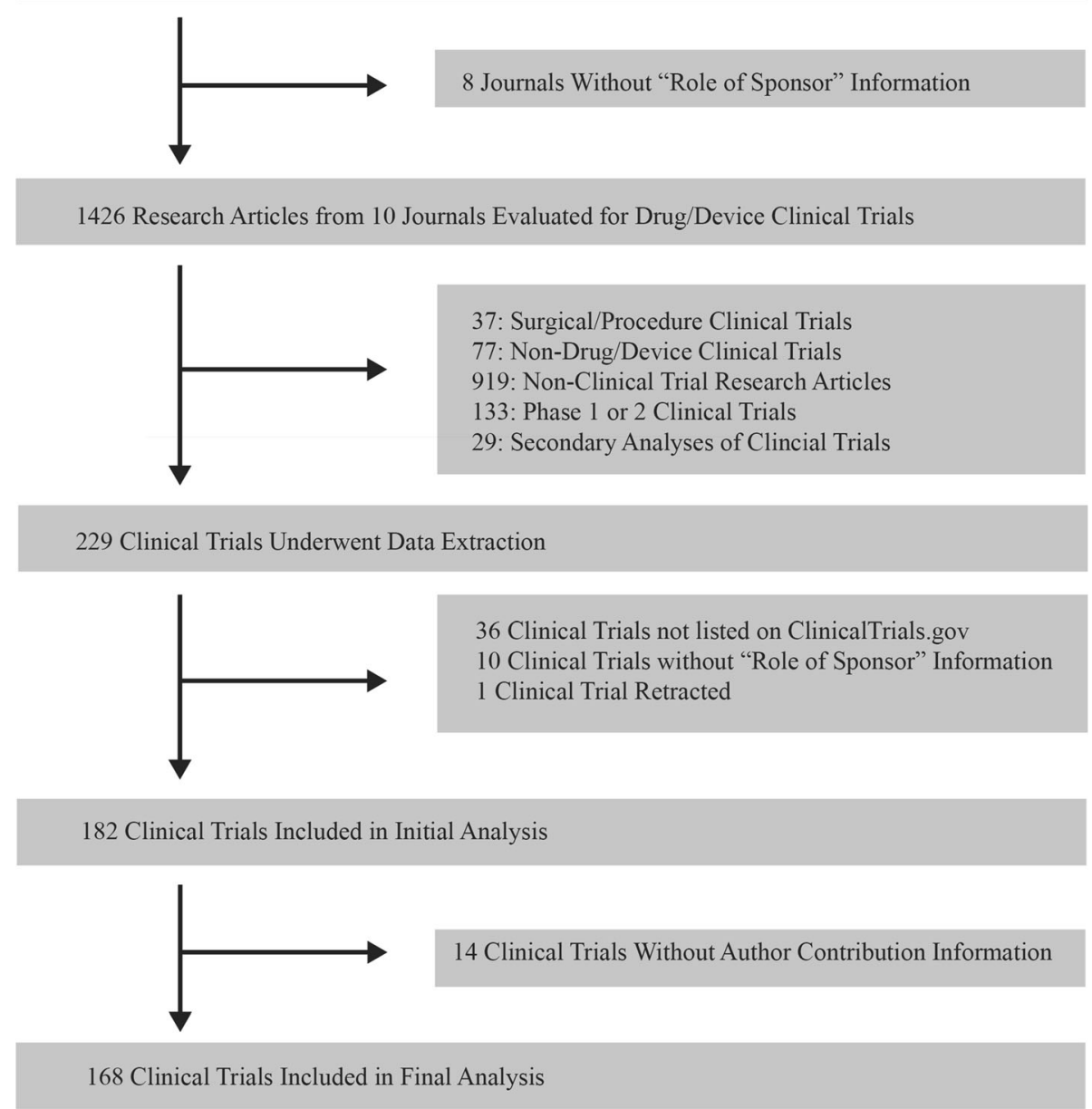

Figure 1. Flow chart for selection of clinical trials included in the study.

of last authors were academically affiliated with no significant differences noted, based on industry funding or collaboration (Table 2). Trials with industry funding with collaboration were more likely to have directly paid either primary author compared to trials with neither industry funding nor collaboration [69.6 (46/76) vs. $2.6(1 / 45) ; p<0.001]$ (Table 2).
Of the ten trials with primary guest authorship, four were from the NEJM, three from Lancet, one from Lancet Oncology, one from Lancet Neurology, and one from Annals of Internal Medicine; in terms of primary clinical specialty, two were Oncology, one Neurology, two Infectious Diseases, and three Endocrinology (Table 3). Three of the ten (30\%) trials

Table 1 Industry Funding and Collaboration Status and Primary Guest Authorship*

\begin{tabular}{|c|c|c|c|c|c|}
\hline & \multirow{2}{*}{ 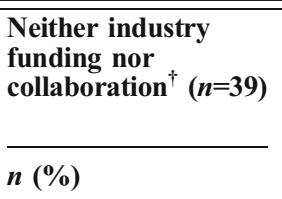 } & \multicolumn{2}{|c|}{$\begin{array}{l}\text { Industry funding } \\
\text { with collaboration } \\
(n=76)\end{array}$} & \multicolumn{2}{|c|}{$\begin{array}{l}\text { Industry funding } \\
\text { without } \\
\text { collaboration } \\
(n=54)\end{array}$} \\
\hline & & $n(\%)$ & $p$ value & $n(\%)$ & $p$ value ${ }^{*}$ \\
\hline Primary guest authorship & $0(0)$ & $10(13.2)$ & 0.02 & $0(0)$ & 1.0 \\
\hline Neither primary author drafted manuscript ${ }^{\S}$ & $0(0)$ & $7(9.2)$ & 0.09 & $0(0)$ & 1.0 \\
\hline Neither primary author designed trial or analyzed/interpretated datall & $0(0)$ & $3(3.9)$ & 0.55 & $0(0)$ & 1.0 \\
\hline
\end{tabular}

* Primary guest authorship defined as neither primary author (first or last) contributing to the either of the following: 1) design of the trial or analysis and interpretation of data or 2) drafting part or all of the manuscript

${ }^{\dagger}$ Reference group

${ }^{t} p$ values calculated by Fisher exact tests compared to reference group

sPrimary authors contributed to either the design or analysis/interpretation of data

"Primary authors contributed to the drafting of the manuscript 
Table 2. Industry Funding and Collaboration Status and Characteristics of Non-Primary Guest Authorship Trials

\begin{tabular}{|c|c|c|c|c|c|}
\hline & \multirow{2}{*}{$\begin{array}{l}\text { Neither industry } \\
\text { funding nor } \\
\text { collaboration } \\
(n=39) \\
n(\%)\end{array}$} & \multicolumn{2}{|c|}{$\begin{array}{l}\text { Industry funding } \\
\text { with collaboration } \\
(n=66)\end{array}$} & \multicolumn{2}{|c|}{$\begin{array}{l}\text { Industry funding } \\
\text { without } \\
\text { collaboration } \\
(n=54)\end{array}$} \\
\hline & & $n(\%)$ & $p$ value $^{\dagger}$ & $n(\%)$ & $p$ value $^{\dagger}$ \\
\hline First author academically affiliated & $39(100)$ & $66(100)$ & 1.0 & $54(100)$ & 1.0 \\
\hline Last author academically affiliated & $37(94.9)$ & $59(89)$ & 0.48 & $48(88.9)$ & 0.46 \\
\hline Either primary author listed as a principal investigator on ClinicalTrials.gov & $33(84.6)$ & $17(25.8)$ & $<0.001$ & $49(90.7)$ & 0.52 \\
\hline Either primary author directly paid by sponsor for trial & $1(2.6)$ & $46(69.6)$ & $<0.001$ & $8(14.8)$ & 0.07 \\
\hline
\end{tabular}

* Reference group

${ }^{\dagger} p$ values calculated by Fisher exact tests compared to reference group

identified a primary author as a principal investigator on ClinicalTrials.gov (Table 3). The first author of all ten primary guest authorship trials $(100 \%)$ had an academic affiliation and the last author had an academic affiliation in eight of the ten trials $(80 \%)$ (Table 2). In all ten trials, either the first or last author was paid directly by the sponsor (Table 2).

\section{DISCUSSION}

We found that $6 \%$ of randomized clinical trials from highimpact journals utilized primary guest authors and that primary guest authorship occurred exclusively among trials with industry funding with collaboration, with a prevalence of $13 \%$. Our results validate the occurrence of primary guest authorship among trials with industry funding with collaboration that was originally revealed by litigation related to rofecoxib. ${ }^{8}$ While we cannot conclude that the sponsors of trials with primary guest authorship specifically recruited academic investigators to serve as primary guest authors (as was the case with rofecoxib), our findings raise this possibility, as all were paid directly for the trial by the sponsor (based on the authors own self-disclosure) and nearly all were academically affiliated.

Table 3. Characteristics of Guest Authorship Trials

\begin{tabular}{ll}
\hline \hline & $\begin{array}{l}\text { Guest } \\
\text { authorship } \\
\text { trials }(\boldsymbol{n}=\mathbf{1 0}) \\
\boldsymbol{n}(\mathbf{\%})\end{array}$ \\
\hline $\begin{array}{l}\text { Clinical specialty } \\
\text { Oncology }\end{array}$ & $2(20)$ \\
$\quad$ Neurology & $1(10)$ \\
Infectious disease & $2(20)$ \\
Endocrinology & $3(30)$ \\
Journal & $3(30)$ \\
NEJM & $4(40)$ \\
Lancet & $1(10)$ \\
Lancet oncology & $1(10)$ \\
$\quad$ Lancet neurology & $1(10)$ \\
Annals of internal medicine & $10(100)$ \\
First author with academic affiliation & $8(80)$ \\
Last author with academic affiliation & $3(70)$ \\
Either primary author listed as a principal \\
investigator on ClinicalTrials.gov
\end{tabular}

Irrespective of the mechanism behind primary guest authorship, the occurrence of this practice among trials with industry funding with collaboration is likely multi-factorial. Industry has been previously reported to remove employee scientist/ physicians with significant contributions from authorship to avoid the appearance of undue commercial influence and to improve the likelihood of publication. ${ }^{11}$ Industry, in general, may also prefer academic primary authors, as readers may discredit study findings when authors are industry employees. ${ }^{12}$ Moreover, beliefs that first and last authors of trials should never be employees of the funding sponsor ${ }^{13}$ or that industry trials should never be published ${ }^{14}$ may push industry toward using guest authors.

We found a lower prevalence of primary guest authorship than what was found in previous guest authorship studies. ${ }^{2-5}$ This result is not surprising for a number of reasons. First, we examined primary guest authorship (only first and last authors) rather than guest authorship among all the listed authors. Second, our definition of primary guest authorship was conservative; we required that neither primary author meet authorship criteria, as opposed to only the first or the last author. We used these approaches, which likely underestimated the presence of guest authorship, in order to detect only guest authorship that would most significantly impact data presentation. Further, we examined clinical trials only because we wished to evaluate publications most likely to influence clinical practice. Lastly, we used author selfdisclosure as opposed to surveys, which may have introduced social desirability bias and led to our underestimating the prevalence of guest authorship.

We found that trials with industry funding with collaboration, including those with guest authorship, were significantly less likely than trials with neither industry funding nor collaboration to have identified the primary authors as the principal investigator(s) on ClinicalTrials.gov, despite no significant difference in the percentage of academically affiliated primary authors between trial types. This finding raises the question of whether the academic primary authors of trials with industry funding with collaboration were truly the principal investigators, especially for the ten trials identified as having primary guest authorship. The finding also suggests that a simple policy shift from journal publishers might further reduce rates of guest authorship: 
ICMJE could consider for publication only clinical trials for which authorship of the final publication is consistent with the principal investigator(s) initially listed on ClinicalTrails.gov (or other online trial registries). Such a shift would be more likely to prevent guest authorship than current policy, which requires inclusion of the principal investigator(s) on ClinicalTrials.gov. ${ }^{7}$

Our study has several limitations. We only included highimpact journals, which may limit the generalizability of our findings. In addition, we excluded journals that do not publish details on the "Role of the Funding Source/Sponsor"; this choice allowed us to determine rates of guest authorship under conditions of optimal transparency, but may limit generalizability or may have led us to underestimate rates of primary guest authorship. Further, information on author contribution was often difficult to find and vaguely written, so our estimates of ghost authorship may include inaccuracies; however, we were intentionally conservative in crediting authors' contributions and are unlikely to have overestimated the prevalence of primary guest authorship. While author contribution data was obtained in duplicate, only one author recorded information on sponsor funding and collaboration, so inaccuracies or bias may have been introduced. However, documentation of funding and collaboration in trial reports is simple and straightforward, so substantial inaccuracy is unlikely. Additionally, although primary guest authorship trials were more prevalent among a few high-impact journals, given the small number of trials, we cannot draw any conclusions about the publishing practices of these specific journals. Lastly, while we hypothesized industry funding and/or involvement may be associated with guest authorship, there may have been confounding risk factors that were not considered.

In conclusion, in this sample of randomized, drug/ device clinical trials in high-impact biomedical journals, we found evidence of primary guest authorship despite increased disclosure requirements by journals. Notably, in our sample, primary guest authorship occurred exclusively among trials with industry funding with collaboration. If the medical and publishing communities wish to reduce guest authorship among such trials, further steps beyond disclosure will be needed.

Acknowledgments: NR collected and analyzed the data. Both authors (NR, DK) participated in the design of the study, interpretation of the results, the writing of the manuscript, and review and approval of the final manuscript. All authors had full access to all the data, including statistical reports and tables, in the study and can take responsibility for the integrity of the data and the accuracy of the data analysis. No funding source was involved in design and conduct of the study; collection, management, analysis, and interpretation of the data; preparation, review, or approval of the manuscript; and decision to submit the manuscript for publication.
Conflict of Interest: The authors declare that they have no conflicts of interest.

Corresponding Author: Nitin Roper, M.D.; Weill Cornell Medical College, 535 East 70th Street, 6th Floor, New York, NY 10021, USA (e-mail: nitinroper@gmail.com).

\section{REFERENCES}

1. PLOS Medicine: Legal Remedies for Medical Ghostwriting: Imposing Fraud Liability on Guest Authors of Ghostwritten Articles. Available at: http:// journals.plos.org/plosmedicine/article?id=10.1371/journal.pmed. 1001070. Accessed March 6, 2015.

2. Mowatt G, Shirran L, Grimshaw JM, et al. Prevalence of honorary and ghost authorship in Cochrane reviews. JAMA. 2002;287(21):2769-71. Available at: http://www.ncbi.nlm.nih.gov/pubmed/12038907. Accessed March 6, 2015.

3. Shapiro DW, Wenger NS, Shapiro MF. The contributions of authors to multiauthored biomedical research papers. JAMA. 1994;271(6):438-42. Available at: http://www.ncbi.nlm.nih.gov/pubmed/8295318. Accessed March 6, 2015.

4. Flanagin A, Carey LA, Fontanarosa PB, et al. Prevalence of articles with honorary authors and ghost authors in peer-reviewed medical journals. JAMA. 1998;280(3):222-4. Available at: http://www.ncbi.nlm.nih.gov/ pubmed/9676661. Accessed March 6, 2015.

5. Wislar JS, Flanagin A, Fontanarosa PB, Deangelis CD. Honorary and ghost authorship in high impact biomedical journals: a cross sectional survey. BMJ. 2011;343:d6128. Available at: http://www.pubmedcentral.nih.gov/ articlerender.fcgi?artid=3202014\&tool=pmcentrez\&rendertype=abstract. Accessed March 6, 2015.

6. International Committee of Medical Journal Editors. ICMJE recommendations for the conduct, reporting, editing and publication of scholarly work in medical journals. 2013. Available at: http://www.icmje.org/recommendations/browse/roles-and-responsibilities/defining-the-role-of-authorsand-contributors.html. Accessed March 6, 2015

7. Section 801 Food and Drug Administration Amendments Act of 2007. Washington, DC Available at: http://www.gpo.gov/fdsys/pkg/PLAW110publ85/pdf/PLAW-110publ85.pdf. Accessed March 6, 2015

8. Ross JS, Hill KP, Egilman DS, Krumholz HM. Guest authorship and ghostwriting in publications related to rofecoxib: a case study of industry documents from rofecoxib litigation. JAMA. 2008;299(15):1800-12. doi:10. 1001/jama.299.15.1800.

9. Roper $\mathbf{N}$, Zhang $\mathbf{N}$, Korenstein $\mathbf{D}$. Industry collaboration and randomized clinical trial design and outcomes. JAMA Intern Med. 2014;174(10):16956. doi:10.1001/jamainternmed.2014.3590.

10. Holmes DR, Hodgson PK, Nishimura RA, Simari RD. Manuscript preparation and publication. Circulation. 2009;120(10):906-13. doi:10. 1161/CIRCULATIONAHA. 107.752782.

11. Hirsch LJ. Conflicts of interest, authorship, and disclosures in industryrelated scientific publications: the tort bar and editorial oversight of medical journals. Mayo Clin Proc. 2009;84(9):811-21. doi:10.1016/ S0025-6196(11)60491-6.

12. Chaudhry S, Schroter S, Smith R, Morris J. Does declaration of competing interests affect readers' perceptions? A randomised trial. BMJ. 2002;325(7377):1391-2. Available at: http://www.pubmedcentral.nih.gov/ articlerender.fcgi?artid $=138516 \&$ tool $=$ pmcentre $\&$ rendertype $=$ abstract . Accessed March 6, 2015

13. Meilof JF, Hylkema MN. The Lancet and advertorials. Lancet. 2009;373(9668): 1004. doi:10.1016/S0140-6736(09)60600-0. author reply 1004-5.

14. Smith R, Gøtzsche PC, Groves T. Should journals stop publishing research funded by the drug industry? BMJ. 2014;348:g171. doi:10. 1136/bmj.g171. 